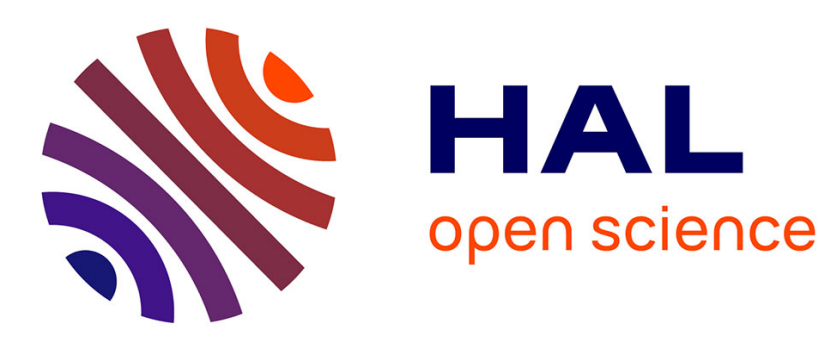

\title{
Coexistence of radio networks using Aloha
}

Bartlomiej Blaszczyszyn, Paul Mühlethaler, Skander Banaouas

\section{To cite this version:}

Bartlomiej Blaszczyszyn, Paul Mühlethaler, Skander Banaouas. Coexistence of radio networks using Aloha. IFIP Wireless Days Conference, Oct 2010, Venice, Italy. 10.1109/WD.2010.5657746 . inria00529904

\section{HAL Id: inria-00529904 https://hal.inria.fr/inria-00529904}

Submitted on 26 Oct 2010

HAL is a multi-disciplinary open access archive for the deposit and dissemination of scientific research documents, whether they are published or not. The documents may come from teaching and research institutions in France or abroad, or from public or private research centers.
L'archive ouverte pluridisciplinaire HAL, est destinée au dépôt et à la diffusion de documents scientifiques de niveau recherche, publiés ou non, émanant des établissements d'enseignement et de recherche français ou étrangers, des laboratoires publics ou privés. 


\section{Coexistence of radio networks using Aloha}

\author{
Bartłomiej Błaszczyszyn \\ INRIA/ENS \\ Paris FRANCE \\ Bartek.Blaszczyszyn@ens.fr
}

\author{
Paul Mühlethaler \\ INRIA Rocquencourt \\ Le Chesnay FRANCE \\ Paul.Muhlethaler@inria.fr
}

\author{
Skander Banaouas \\ INRIA Rocquencourt \\ Le Chesnay FRANCE \\ Iskander.Banaouas@inria.fr
}

\begin{abstract}
In this paper we use an analytical model to study how two radio networks : a primary network and a secondary network can coexist within the same area. We use a very simple protocol, Aloha, for both radio networks, and tools borrowed from stochastic geometry to model the performance of our two networks. We show that the primary network and the secondary network can adapt their transmission parameters simultaneously to achieve the following goal : the primary network maintains its performance with a maximum and fixed degradation whereas the secondary network maximizes its transmission throughput. In practice this involves the primary network adapting its transmission power and the secondary network its transmission probability. We also study the gain in performance when the secondary network nodes only transmit when their receivers are at minimum distance from any transmitter nodes in the primary network (constrained distance deployment).

We obtain the following result : when there is no constraint used for the secondary network (free deployment), it can offer a low (but not completely negligible) density of successful transmissions when the capture threshold in primary network $T_{1}$ is small. If we optimize the density of successful transmissions for the selected nodes in the secondary network which are transmitting to receivers at a minimum distance from any node in the primary network then the per node throughput for these selected nodes can be very significantly increased. However with this optimization the total network throughput for the secondary network is also slightly reduced. If we use a constrained distance deployment for the secondary network (only nodes whose receivers at a minimum distance from the primary network) the per node throughput can be very significantly increased and the total network throughput for the secondary network is increased.
\end{abstract}

Index Terms-Ad hoc networks, slotted Aloha, transmission probability, Cognitive radio, Poisson point process, shot-noise, SINR, stochastic geometry

\section{INTRODUCTION}

The aim of this paper is to show that it is possible to share the same medium with a very simple Medium Access Control mechanism (MAC): Aloha. We obtain this result using simple models based on stochastic geometry. More precisely we show that if we adapt the transmission power of the primary and secondary networks and if we also use different CDMA codes, the performance of the primary network can be maintained while the secondary network can provide its users with an acceptable service for short range communication.

\section{A. Related Work}

Most of the studies which deal with cognitive radio try to optimize criteria based on the Signal over Interference-andNoise Ratio (SINR) [4, 6, 7, 9]. These papers aim to achieve optimizations using classical techniques : relaxation, convex optimization, etc. But it is difficult to apply these results in decentralized systems. However very few of these studies consider real medium access mechanisms, the focus being on adapting the power of a node or selecting a given channel to optimize a global (or local) criterion. In nearly all the papers dealing with cognitive radio and resource allocation, the temporal aspect is not addressed: the decisions that the algorithms provide are supposed to hold for long periods. Similarly the spatial aspect of the problem is seldom considered even though new results allow the interference to be exactly computed under a few but rather general assumptions [2, 3].

In this paper we study the coexistence of two networks which use slotted Aloha as a MAC protocol. We show that we can extend an existing model to compute the probability of successful transmissions in both networks. We study how the two networks can coexist and show that we can have reasonable performances if the primary network adapts its transmission power and the secondary network adapts its transmission probability. We also show that if the secondary network accepts a constraint on its deployment, it can greatly improve its performance. However in this case the primary network must increase its transmission power to maintain its performance.

\section{B. Modeling Assumptions}

The primary network is allowed to use a much greater transmission power than the secondary network. The primary network also uses CDMA codes. Since we use slotted Aloha, we can assume the primary network to be precisely synchronized. Thus, with CDMA codes we can assume that the Signal over Interference Ratio (SIR) $T_{1}$ required to accept a packet is small compared to 1 , whereas a typical value for SIR without using CDMA codes is $T_{1}=10$.

\section{Modeling And Problem Formulation}

\section{A. Two Network Model}

1) Primary and Secondary Network Nodes: In this paper we consider two coexisting networks called respectively primary and secondary network, each of which is represented by the so called Poisson Bipole model proposed in [2]; see also [1, Chapter 16]. The nodes of these two networks are distributed on the infinite plane according to two independent, homogeneous, planar Poisson point processes (P.p.p.) of intensity $\lambda_{1}$ and $\lambda_{2}$ nodes per unit surface area (say per square meter) respectively. Each node of each of these two networks is wishing to transmit a packet to its own dedicated receiver located within a distance $r_{a}, a=1,2$, for the primary and secondary networks, respectively. These receivers are not part of the Poisson point processes. Despite its drawbacks, mostly related to the modeling of the locations of receivers, 
this Poisson Bipole network model is reasonable, convenient and seems to be widely accepted (cf $[5,8]$ ).

Using the formalism of the theory of point processes, we can say that a joint snapshot of primary and secondary network nodes can be represented by two independently marked Poisson point processes (P.p.p) $\left(\tilde{\Phi}_{1}, \tilde{\Phi}_{2}\right)$ with $\widetilde{\Phi}=\left\{\left(X_{i}^{a}, y_{i}^{a}\right)\right\}$, $a=1,2$, where the locations of nodes $\Phi^{a}=\left\{X_{i}^{a}\right\}$ form a homogeneous P.p.p. of intensity of $\lambda^{a}$ and where the marks $y_{i}^{a}$ denote the locations of the receivers for nodes $X_{i}^{a}$. We assume here that one receiver is associated with only one transmitter and that, given $\Phi_{1}, \Phi_{2}$, the vectors $\left\{X_{i}^{a}-y_{i}^{a}\right\}$ are i.i.d with $\left|X_{i}^{a}-y_{i}^{a}\right|=r_{a}$.

2) Wireless Channel Model: We assume that whenever node $X \in \Phi^{a}(a=1,2)$ transmits a packet with some power $P$, the signal that is propagated and reaches any given location $y$ on the plane with power equal to $P F / l(|X-y|)$, where $|\cdot|$ denotes the Euclidean distance on the plane, $l(\cdot)$ is some deterministic path-loss function of the distance and $F$ is a nonnegative random factor of unit mean representing the variations in the received power due to channel fading.

An important special case, which is our default assumption in this paper, consists in taking a path-loss function of the form

$$
l(u)=(A u)^{\beta} \quad \text { for } A>0 \text { and } \beta>2 .
$$

Regarding the distribution of the random variable $F$, called for simplicity fading, in this paper we will consider only the special case of Rayleigh fading that corresponds to the exponential distribution of $F$, whose mean is assumed to be $\mathbf{E}[F]=1$. More precisely, we will assume that for any pair: emitter $X$, location $y$ of a potential receiver, an independent copy $F=F_{X, y}$ is used to model the fading throughout the transmission of this packet.

3) Slotted Aloha MAC: We assume that both networks use the slotted Aloha MAC scheme and are synchronized to the same time slots (this assumption can be easily relaxed later) but are however tuned differently. By different tuning we mean different emitted power and different medium access probability. More precisely, let us assume that each node of the network $a(a=1,2)$, at a tagged time slot, independently tosses a coin with some bias $p_{a}$ and sends its packet using power $P_{a}$ in this time slot if the outcome is heads and does not transmit otherwise.

4) Successful Transmission: It is natural to assume that transmitter $X_{i}^{a}$, successfully transmits a given packet to its receiver $y_{i}^{a}$ if

$$
\mathrm{SIR}=\frac{P_{a} F / l\left(\left|X_{i}^{a}-y_{i}^{a}\right|\right)}{\bar{I}} \geq T_{a},
$$

where $P_{a}$ is the emitted signal power, $T_{a}$ is some signalto-interference (SIR) threshold for network $a$ and where $\vec{I}$ is the total interference suffered by the receiver $y_{i}^{a}$. In the two-network model this total interference can be naturally expressed as the sum $\bar{I}=I_{1}+I_{2}$ of the interferences created by the concurrent transmissions of the primary and secondary networks . For $(a, b) \in\{1,2\}^{2}$

$$
I_{b}=\sum_{X_{j}^{b} \in \Phi^{b}, X_{j}^{b} \neq X_{i}^{a}} P_{b} F_{X_{j}^{b}, y_{i}^{a}} / l\left(\left|X_{j}^{b}-y_{i}^{a}\right|\right) \mathbb{I}\left(X_{j}^{b} \text { transmits }\right) .
$$

Note that taking (2.2) as the successful transmission condition, we ignore the external noise. This is a reasonable assumption if the noise is significantly smaller than the mean interference power.

\section{B. Problem Formulation}

We denote by $p_{\text {cov }}^{a}, a=1,2$ the probability of successful transmissions (coverage probability) in a given time slot by a typical node of the network $a$, provided it is scheduled for the transmission by the respective Aloha MAC. Consequently, the density of successful transmissions (defined as the expected number of such transmissions per node of the respective network $a$ ) can be expressed as $d_{a}=p_{a} p_{c o v}^{a}$.

The main objective of this paper is to study the maximization of the density of successful transmissions in the secondary network given a constraint (a lower bound) on the coverage probability in the primary network. This optimization will be achieved by some tuning of the power $P_{1}$ of the primary users and the Aloha MAC transmission probability $p_{2}$ used by the secondary users

1) Optimization without Deployment Control: We assume that the Aloha MAC parameter $p_{1}$ is fixed. Under these assumptions, $p_{\text {cov }}^{a}\left(P_{1}, p_{2}\right)$, and $d_{a}\left(P_{1}, p_{2}\right)$ denote respectively the coverage probability and the density of successful transmissions in the network $a=1,2$, when the primary users emit with power $P_{1}$ and the MAC probability in the secondary network is fixed to $p_{2}$. Note that when the secondary users do not emit at all $\left(p_{2}=0\right)$, we have only the primary-network interference $I=I_{1}$ and thus the SIR condition (2.2) for $a=1$ is invariant with respect to the power $P_{1}$ used by the primary nodes. Consequently $p_{\max . c o v}^{1}:=p_{\text {cov }}^{1}(1,0)$ is the (maximal) coverage probability that the primary users can achieve when no secondary nodes are allowed to transmit.

The aforementioned optimization problem can now be formalized as follows. For a given $\delta>0$, representing an acceptable degradation of the coverage probability in the primary network, we want to find the maximal density of successful transmissions $\bar{d}_{2}$ that the secondary network can achieve

$$
\begin{array}{r}
\bar{d}_{2}:=\max _{0 \leq p_{2} \leq 1}\left\{d_{2}\left(P_{1}, p_{2}\right): p_{\text {cov }}^{1}\left(P_{1}, p_{2}\right) \geq(1-\delta) p_{\text {max.cov }}^{1}\right. \\
\text { for some } \left.P_{2}<\infty\right\}
\end{array}
$$

and the respective optimal tuning $\bar{p}_{2}$ of the Aloha MAC probability $p_{2}$ of the secondary network for which the maximum (2.4) is attained. We will denote by $\bar{P}_{1}$ the minimal power the primary nodes have to use in order to maintain their coverage probability at the required level when the secondary users implement $\bar{p}_{2}$. Thus $\bar{P}_{1}$ is such that $p_{\text {cov }}^{1}\left(\bar{P}_{1}, \bar{p}_{2}\right)=$ $(1-\delta) p_{\max . c o v}^{1}$.

2) Secondary Network with Exclusion Zones: Besides the optimization of the secondary network described above, we 
study if the obtained density of successful communications can be further improved by controlling the deployment of the secondary network nodes. We investigate the following simple geographic inhibition rule on the deployment of the secondary network: only transmitters in the secondary network whose receivers are closer than some given separation distance $R$ to any of transmitter in the primary network can transmit.

\section{Problem AnAlysis}

The optimization problem formulated in the previous section can be explicitly solved for an unconstrained deployment of the secondary network. In what follows we will first present this solution. Then we show some approximations of the solution when the primary and secondary network users are geographically separated.

\section{A. Optimization without Deployment Control}

The problem consists in an optimal tuning of the Aloha MAC probability of the secondary users in order to maximize their density of successful transmissions, while the primary users react by fixing their transmission power so as not to decrease their coverage probability by more than $\delta \times 100 \%$. The following result gives the explicit solution in the case of the (unconstrained) Poisson deployment of the secondary network.

Proposition 3.1: Assume the unconstrained Poisson distribution of the secondary nodes. Then the maximal density of successful transmission $\bar{d}_{2}$ in this network, defined in (2.4), is achieved for

$$
\bar{p}_{2}=\frac{1}{r_{2}^{2} T_{2}^{2 / \beta} K(\beta) \lambda_{2}\left(1+\frac{\lambda_{1} p_{1} r_{1}^{2} T_{1}^{2 / \beta} K(\beta)}{-\log (1-\delta)}\right)}
$$

and

$$
\bar{P}_{1}=\frac{P_{2} T_{1}}{T_{2}}\left(\frac{r_{1}}{r_{2}}\right)^{\beta}\left(\lambda_{1} p_{1} r_{1}^{2}-\log (1-\delta) T_{1}^{2 / \beta} K(\beta)\right)
$$

where $K(\beta)$ is a constant given by:

$$
K(\beta)=2 \pi \Gamma(2 / \beta) \Gamma(1-2 / \beta) / \beta .
$$

Proof: Using the known explicit result for the coverage probability in the Poisson Bipole network (see e.g. [3] or [1, Chapter 16]) and the fact that the interferences $I_{1}$ and $I_{2}$ created by the primary and secondary networks (for some given tuning of these networks) are independent, we obtain the following probabilities of successful transmission $p_{\text {cov }}^{a}=p_{\text {cov }}^{a}\left(P_{1}, p_{2}\right)$ :

$$
\begin{aligned}
p_{\text {cov }}^{1}= & \exp \left(-r_{1}^{2} T_{1}^{2 / \beta} K(\beta) \lambda_{1} p_{1}\right) \\
& \times \exp \left(-r_{1}^{2}\left(\frac{T_{1} P_{2}}{P_{1}}\right)^{2 / \beta} K(\beta) \lambda_{2} p_{2}\right) \\
p_{\text {cov }}^{2}= & \exp \left(-r_{2}^{2} T_{2}^{2 / \beta} K(\beta) \lambda_{2} p_{2}\right) \\
\times & \exp \left(-r_{2}^{2}\left(\frac{T_{2} P_{1}}{P_{2}}\right)^{2 / \beta} K(\beta) \lambda_{1} p_{1}\right) .
\end{aligned}
$$

Fixing $p_{2}$ and solving $p_{\text {cov }}^{1}\left(\bar{P}_{1}, p_{2}\right)=(1-\delta) p_{\text {cov }}^{1}(1,0)$ in $\bar{P}_{1}$ we obtain

$$
\bar{P}_{1}=T_{1}\left(\frac{r_{1}^{2} \lambda_{2} K(\beta) p_{2}}{-\log (1-\delta)}\right)^{\beta / 2} P_{2} .
$$

Using the above value of $\bar{P}_{1}$ in $d_{2}\left(\bar{P}_{1}, p_{2}\right)=p_{2} p_{\operatorname{cov}}^{2}\left(\bar{P}_{1}, p_{2}\right)$ and maximizing this expression in $p_{2}$, we obtain (3.5). Finally inserting this expression in (3.10) we obtain (3.6).

\section{B. Optimization of the selected secondary users.}

Now we turn our attention in the optimization of the density $d_{2}^{\prime}$ of successful transmissions (per node) of secondary users taking into account only users $X_{i}^{2} \in \Phi_{2}$ whose receivers are such that $\left|y_{i}^{2}-X_{j}^{1}\right| \geq R$ for all $X_{j}^{1} \in \Phi_{1}$. But in contrast to the situation depicted in Section III-C the all the secondary users still transmit.

For such secondary users, the corresponding conditional interference produced by the primary users can be evaluated as in Figure 1. The interferers in the primary network are necessarily in the shaded area. Thus the coverage area for these secondary users can be simply computed; the shot-noise is just truncated.

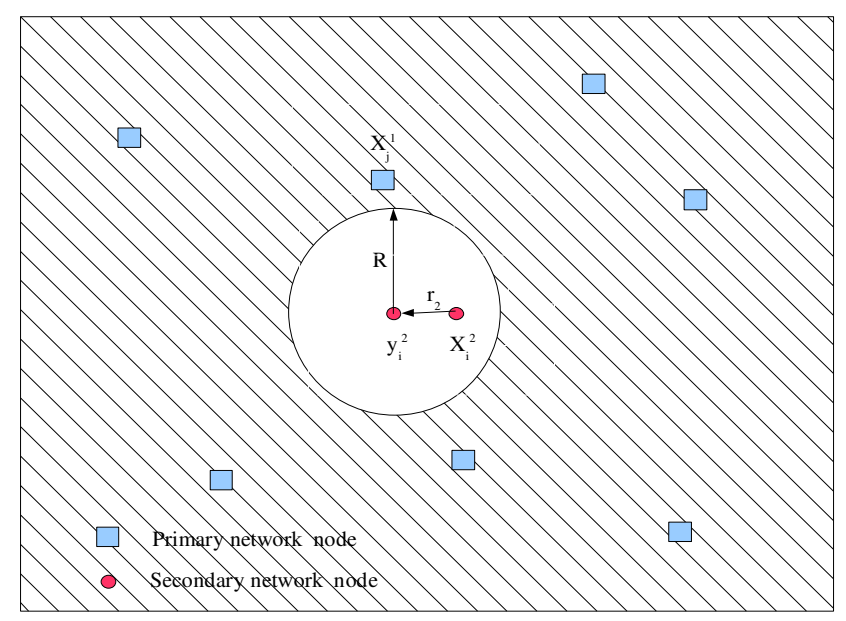

Fig. 1. Approximation to compute the Laplace transform of the shot-noise created by the primary network to the secondary network. The shaded area is where nodes of the primary network can be located.

The coverage probability for the selected nodes in the secondary network is $p_{\text {cov }}^{\prime 2}$ given below:

$$
\begin{aligned}
p_{\text {cov }}^{\prime 2}\left(P_{1}, p_{2}\right) & =\exp \left(-r_{2}^{2}\left(\frac{T_{2} P_{1}}{P_{2}}\right)^{\frac{2}{\beta}} K\left(\beta, \frac{R}{r_{2}}\left(\frac{P_{2}}{P_{1} T_{2}}\right)^{\frac{1}{\beta}}\right) \lambda_{1} p_{1}\right) \\
& \times \exp \left(-r_{2}^{2} T_{2}^{2 / \beta} K(\beta) \lambda_{2} p_{2}\right)
\end{aligned}
$$

where

$$
K(\beta, a)=\int_{a}^{\infty} \frac{x}{x^{\beta}+1} \mathrm{~d} x .
$$

The density $d_{2}^{\prime}$ of successful transmissions (per node) for secondary users whose receivers are at least $R$ meters away from any primary user is given by the following equation:

$$
d_{2}^{\prime}\left(P_{1}, p_{2}\right)=p_{2} p_{\text {cov }}^{\prime 2}\left(P_{1}, p_{2}\right)
$$


This density will be evaluated numerically in Section IV where we study the benefit for the nodes in the secondary network whose receivers are at a distance of at least $R$ from any nodes in the primary network.

\section{Secondary network deployed outside exclusion zones}

We now study the performance when the secondary network is deployed outside exclusion zones as described in Section II-B2. We consider the nodes in the secondary network whose receiver nodes are at distance of at least $R$ from any nodes in the primary network as in Section III-B. But in contrast, we consider that only these nodes in the secondary network are allowed to transmit.

The spatial intensity of the secondary users is equal to $\lambda_{2} e^{-\pi \lambda_{1} R^{2}}$, where the exponential expression corresponds to the mean fraction of the surface of the plane not covered by the spherical Boolean model of intensity $\lambda_{1}$ and disk radius $R$ (i.e. one minus the so called volume fraction of the Boolean model).

In the following we assume that

- the interferences produced by the nodes in the primary and secondary network are independent,

- the interference produced by the nodes of the secondary network is equivalent to the interference produced by a Poisson process of spatial intensity $\lambda_{2} e^{-\pi \lambda_{1} R^{2}}$.

These assumptions are validated in Section IV by comparing the results of the model with those of simulations. We obtain the following probabilities of successful transmission $p_{c o v}^{\prime \prime a}=$ $p_{\text {cov }}^{\prime \prime a}\left(P_{1}, p_{2}\right)$

$$
\begin{aligned}
p_{\text {cov }}^{\prime \prime 1}= & \exp \left(-r_{1}^{2} T_{1}^{2 / \beta} K(\beta) \lambda_{1} p_{1}\right) \\
\times & \exp \left(-r_{1}^{2}\left(\frac{T_{1} P_{2}}{P_{1}}\right)^{2 / \beta} K(\beta) e^{-\pi \lambda_{1} R^{2}} \lambda_{2} p_{2}\right) \\
p_{\text {cov }}^{\prime \prime 2}= & \quad \exp \left(-r_{2}^{2}\left(\frac{T_{2} P_{1}}{P_{2}}\right)^{2 / \beta} K\left(\beta, \frac{R}{r_{2}}\left(\frac{P_{2}}{P_{1} T_{2}}\right)^{1 / \beta}\right) \lambda_{1} p_{1}\right) \\
\times & \exp \left(-r_{2}^{2} T_{2}^{2 / \beta} K(\beta) \lambda_{2} p_{2} e^{-\pi \lambda_{1} R^{2}}\right) .
\end{aligned}
$$

Fixing $p_{2}$ and solving $p_{\text {cov }}^{\prime \prime 1}\left(\hat{P}_{1}, p_{2}\right)=(1-\delta) p_{\text {cov }}^{\prime \prime 1}(1,0)$ in $\hat{P}_{1}$ we obtain:

$$
\hat{P}_{1}=P_{2} T_{1}\left(\frac{r_{1}^{2} \lambda_{2} K(\beta) p_{2} e^{-\pi \lambda_{1} R^{2}}}{-\log (1-\delta)}\right)^{\beta / 2} .
$$

If we use $P_{1}=\hat{P}_{1}$ in $p_{\text {cov }}^{\prime \prime 2}$ we can numerically optimize $d_{2}^{\prime \prime}=$ $p_{2} p_{\text {cov }}^{\prime \prime 2}$ versus the transmission probability in the secondary network $p_{2}$. This optimization is presented in Section IV-C and compared with simulation results.

\section{RESUlts}

We use $\beta=4$ and the following parameters for the primary and secondary network:

- $\lambda_{1}=0.0001, p_{1}=1, r_{1}=100 \mathrm{~m}$ and $T_{1}$ varies from 0.01 to 10 ,

- $\lambda_{2}=0.01, r_{2}=10 \mathrm{~m}$ and $T_{2}=10$. We adopt $P_{2}=$ $10 \mathrm{~mW}$.
To control the performance reduction of the primary network we use $\delta=0.05$. We set $R=55 \mathrm{~m}$ when we constrain the deployment of the secondary network.

As stated in Section II-B, the primary users compute $P_{1}$ in order to maintain their reception probability and the secondary users optimize their transmission probability taking into account the transmission power $P_{1}$ computed by the primary users.

\section{A. Optimization without deployment Control}

First, to validate our model, we compute the (per node) density of successful transmissions for the secondary network versus the transmission probability $p_{2}$. The comparison beween our model and the simulations are given in Figure 2. We note a very good matching between the model and the simulations. The maximum density of successful transmissions per node is 0.0022 which is roughly a tenth of the maximum density of successful transmissions of the secondary network when this network operates alone, i.e., 0.024 .

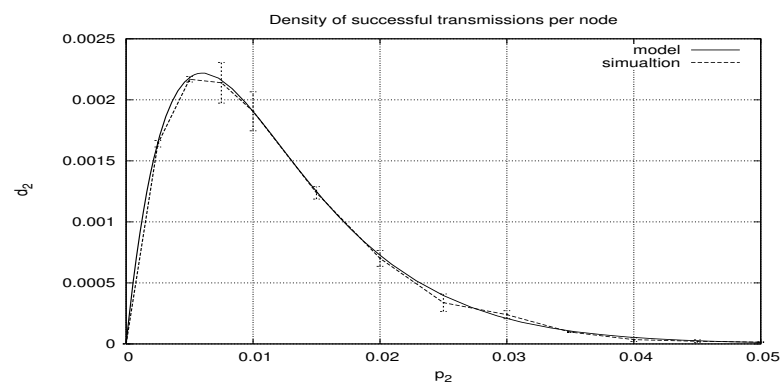

Fig. 2. Density of successful transmissions for the secondary network versus transmission probability $p_{2}$. Comparison between the analytical model and simulations

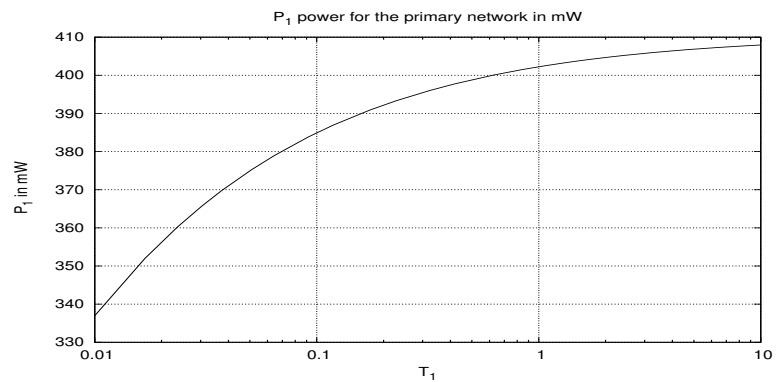

Fig. 3. Required power $P_{1}$ for the primary network versus the capture threshold $T_{1}$ when the secondary network is freely deployed

In Figure 3, we compute the power that the primary network must use to keep its reception probability greater than $1-\delta$ times the reception probability of the primary network when it operates without the secondary network. This power is computed versus the capture threshold $T_{1}$ in the primary network. We observe that the increase in this power remains limited. However in Figure 4, we compute the maximun density of successful transmissions per node that the secondary network can obtain versus the capture threshold $T_{1}$. This density of 
successful transmissions per node could be acceptable for $T_{1}=0.01$, it is roughly one tenth the value of the density of successful transmissions per node (i.e. 0.024) when there is no primary network. When $T_{1}$ becomes larger, the density of throughput that the secondary network exhibits tends to be very small.

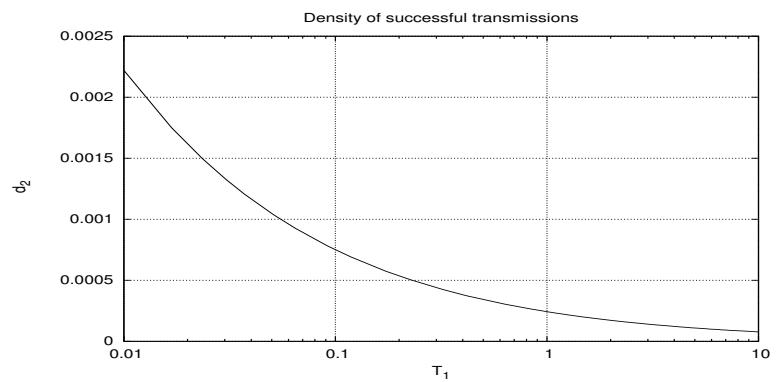

Fig. 4. Density of successful transmissions for the secondary network versus the capture threshold $T_{1}$ when the secondary network is freely deployed

\section{B. Optimization of the selected secondary users}

In Figure 5, we compute the density of successful transmissions (per node) for the selected secondary users versus the transmission probability $p_{2}$. The primary network still computes $P_{1}$ to maintain its reception probability. The maximum of this density of successful transmissions is 0.0042 reached for $p_{2}=0.0078$. We have run simulations which confirm this maximum value. We observe that this density of successful transmissions is double for the selected secondary users; in the default configuration this density of successful transmissions is around 0.002 as shown in Figure 4. However if we compute the total throughput obtained by the selected secondary users, we find a total throughput of $0.0042 \times \lambda_{2} \exp \left(-\pi \lambda_{1} R^{2}\right)=$ 0.000016 transmissions per surface unit. This value is slightly smaller than the total throughput 0.000022 obtained within the whole network in the default configuration.

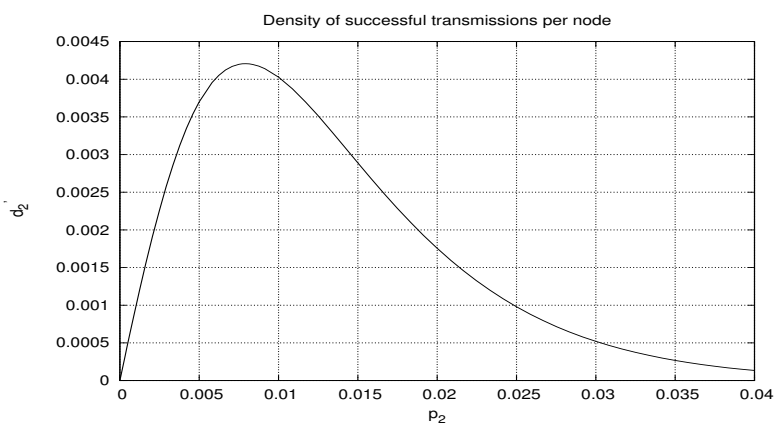

Fig. 5. Density of successful transmissions for the selected nodes in the secondary network versus $p_{2}$

\section{Secondary network deployed outside exclusion zones}

We use equation 3.13 to we compute the density of successful transmissions (per node) when the secondary network is deployed outside exclusion zones. The maximum of this (per node) density of successful transmissions is 0.011 and it is reached for $p_{2}=0.021$. Simulations confirm this value and justify the assumptions used in the analytical model. We notice that the (per node) density of successful transmissions is roughly half the (per node) density of successful transmissions when the secondary network operates alone. The total network throughput is $0.011 \times \lambda_{2} \exp \left(-\pi \lambda_{1} R^{2}\right)=0.000042$ transmissions per surface unit. Thus the constraint on its deployment also allows the secondary network to obtain roughly twice the total throughput than in the free deployment. The power $P_{1}$ that the primary network must use to maintain its reception probability is $550 \mathrm{~mW}$ which is an acceptable value.

\section{Other optimizations}

The model presented in this paper allows many other optimizations to be conducted. They are not presented in this paper for reasons of space.

\section{CONCLUSION}

We have shown that simple models can predict the behaviour of two coexisting networks using Aloha as a MAC protocol. The primary network can maintain its performance by using CDMA to reach a small capture threshold and adapting its transmission power. If the secondary network only optimizes its transmission probability, it obtains a small density of successful transmissions. If the secondary network is deployed outside exclusion zones, it can greatly improve the per node density of successful transmissions and also its total throughput.

\section{REFERENCES}

[1] F. Baccelli and B. Błaszczyszyn. Stochastic Geometry and Wireless Networks, Volume II - Applications. Foundations and Trends in Networking. NoW Publishers, 2009. 209 pages.

[2] F. Baccelli, B. Blaszczyszyn, and P. Mühlethaler. An Aloha Protocol for Multihop Mobile Wireless Networks. In Proceedings of the Allerton Conference, University of Illinois, Urbana Champaign, November 2003. and IEEE Transactions on Information Theory, 52(2):421-436, 2006.

[3] F. Baccelli, B. Błaszczyszyn, and P. Mühlethaler. Stochastic analysis of spatial and opportunistic Aloha. IEEE JSAC, special issue on Stochastic Geometry and Random Graphs for Wireless Networks, 2009. to appear.

[4] Mung Chiang, Chee Wei Tan, Daniel P. Palomar, and David Julian. Power control by geometric programming. IEEE Trans. on Wireless Communications, 5:289-313, Nov 28-Dec 2, 2005.

[5] R. K. Ganti and M. Haenggi. Spatial and Temporal Correlation of the Interference in ALOHA Ad Hoc Networks. In IEEE Communication Letters, September 2009.

[6] Zhi-Quan Luo and Jong-Shi Pang. Analysis of iterative waterfilling algorithm for multiuser power control in digital subscriber lines. EURASIP J. Appl. Signal Process., 2006:80-80, january. ISSN 1110-8657. doi: http://dx.doi.org/10.1155/ASP/2006/24012.

[7] C. W. Tan, D. P. Palomar, and M. Chiang. Solving nonconvex power control problems in wireless networks: Low sir regime and distributed algorithms. In Proc. IEEE GLOBECOM, St. Louis, MO, pages 794-802, 2005.

[8] Steven P. Weber, Xiangying Yang, Jeffrey G. Andrews, and Gustavo de Veciana. Transmission Capacity of Wireless Ad Hoc Networks With Outage Constraints. In IEEE Transactions On Information Theory, Vol. 51, No. 12, December 2005.

[9] W. Yu and R. Lui. Dual methods for nonconvex spectrum optimization of multicarrier systems. IEEE Trans. Communications, pages 1310-1322, 2006. 